\title{
Le chanoyu, cérémonie japonaise traditionnelle du thé, comme exemple d'éducation à la présence
}

Pascal Bouchez, Université de Lille Nord de France, UVHC, DeVisU, Valenciennes, France

\section{Introduction}

Il est coutumier d'entendre dire encore aujourd'hui qu'on ne peut vraiment connaitre le savoir-vivre et l'esprit japonais qu'en ayant pratiqué la cérémonie du thé. Le chanoyu, littéralement « eau chaude pour le thé », décontenance pourtant beaucoup d'occidentaux, et pas uniquement des touristes étrangers fréquemment confrontés à un rituel technique esthétisant dénué de ses fondements authentiques. Le souvenir le plus commun pour une personne non avertie se résume bien souvent à un inconfort physique ainsi qu'à un vif agacement intime en réaction à la lenteur et à la codification extrêmes du dispositif.

Pour analyser ce cérémoniel, en l'absence d'une approche de type ethnométhodologique, la perspective adoptée - à visée exploratoire - s'attache à cerner la réalité intersubjective, son «sens vécu» (Maisonneuve 12), mais plus encore, dans une visée systémique, la cognition incarnée (Varela, Thompson et Rosch 207-247) et la construction orchestrale (Birdwhistell) du sens qui émerge des interactions ritualisées. Ne seront donc pas utilisées ici les multiples formes classificatrices et interprétatives du champ étendu de la pensée ritologique, ni même les analyses détaillées de séquences gestuelles de «microsrituels », comme par exemple celui du salut lors d'une rencontre de judo (Bui-Xuân et Ruffié).

Après avoir présenté le dispositif communicationnel dans sa structure élémentaire, dans l'explicitation de ses principes traditionnels de réalisation, dans son intentionnalité, et dans ses composantes esthétiques, nous nous attacherons à la notion de technique mise en jeu. Nous montrerons à travers l'exemple voisin des arts martiaux qu'elle s'inscrit dans un paradigme «étendu » très éloigné de celui qui fonde la rationalité occidentale aujourd'hui mondialisée. La compréhension du sens partagé - intensification de la présence - qui émerge de la succession des ritèmes du chanoyu fera l'objet de la partie suivante. Elle rendra possible une interrogation conclusive sur l'urgente nécessité selon nous de l'élaboration d'outils didactiques d' « éducation à la présence », à même d'optimiser les configurations et les usages des TICE en prenant mieux en compte les différents niveaux de réalité mis en jeu dans la corporéité, « impensé » que le chanoyu a le grand mérite de souligner. 


\section{1- La cérémonie japonaise du thé}

\section{1- Présentation}

La cérémonie telle que nous la connaissons de nos jours est le résultat d'une lente maturation, dans laquelle «le taoïsme a fourni la base des idéaux esthétiques, le zen les a rendus pratiques »(Okakura 47). C'est aux quinzième et seizième siècles que le zen et sa perception singulière du monde s'approprient véritablement le chanoyu comme moyen d'action, donnant à la « Voie du thé » (chadô), avec Sen no Rikyu (1522-1591) en particulier, toute son originalité.

La cérémonie du thé comprend normalement une première partie au cours de laquelle on sert une collation légère (le kaiseki), une courte pause (nakadachi), la partie principale de la cérémonie (goza-iri), avec le service du koicha ou thé épais, enfin le service de l'usucha, ou thé léger. L'ensemble de la cérémonie dure environ quatre heures mais il est courant de se contenter de l'usucha, la forme abrégée, qui prend moins d'une heure. Il est ici hors de propos de vouloir décrire l'étiquette extrêmement minutieuse et particulièrement formalisée d'une cérémonie du thé traditionnelle ${ }^{1}$. Le protocole repose cependant sur des principes fort simples.

\section{2- Principes directeurs de la "Voie du thé"}

Selon Sen Sôshitsu XV, Hounsai (quinzième descendant de Sen no Rikyu), et jusqu'en 2002 maitre de la principale lignée Urasenke, «la Voie du thé est une façon d'enseigner, à travers le geste de servir et celui de recevoir un bol de thé, une manière de vivre $\gg($ Sen 47$)$.

Les quatre principes directeurs transmis par la Tradition sont les suivants: «Harmonie (wa), Respect (kei), Pureté (sei), Sérénité (jaku)». Wa signifie paix, unité, harmonie, mais aussi action d'adoucir ou de calmer. C'est l'aptitude inconditionnelle à établir à chaque instant l'ordre et la paix en soi et dans sa relation au monde. Kei suggère le respect, l'honneur et la vénération, mais aussi la distanciation. Le respect donne sa structure protocolaire à la cérémonie, mais il manifeste avant tout la capacité de comprendre et d'accepter les autres dans toutes leurs différences. Chacun est de dignité égale dans la pièce de thé, les hiérarchies sociales sont suspendues. Ce respect s'étend aux ustensiles, quelles que soient leurs origines, et à la vie elle-même. Sei désigne l'action de purifier, de nettoyer. C'est en fait l'essence de l'entrainement au chadô, la Voie du thé : l'ascèse disciplinée au quotidien. Pas un grain de poussière ne doit être oublié. Mais en-deçà des formes extérieures, la pureté vise la capacité initiale de se traiter et de traiter les autres avec un cœur ouvert, c'est-à-dire, en premier lieu, dans un état de «non-défensivité » ${ }^{2}$. Jaku, enfin, veut dire silencieux, doux, 
immobile : «juste tranquille ». La sérénité est le point dans l'ascèse bien menée où le pratiquant se retrouve désengagé d'une perception égocentrique de lui-même. À ce moment là, il est dit de manière sobrement allusive que les « possibilités infinies de la vie » peuvent se voir réalisées.

La ligne de conduite essentielle à respecter dans le chanoyu est aussi contenue dans sept « règles » d'esprit zen, énoncées par Sen no Rykiu. "Fais un délicieux bol de thé » signifie notamment que le thé ne vaut pas grand chose sans l'attitude intérieure des quatre grands principes évoqués précédemment. " Dispose le charbon de bois de façon à chauffer l'eau » témoigne du degré d'accomplissement de l'hôte : «L'idéal de la Cérémonie du Thé, c'est "un mouvement sans faille", c'est-à-dire sans perte d'énergie. Cela s'exprime jusque dans les plus infimes détails, y compris l'arrangement du charbon dans le braséro en fer » (Reed 223) ${ }^{3}$. «Arrange les fleurs comme elles sont dans les champs » requiert une sensibilité esthétique frémissante et imprévisible. "En été, évoque la fraicheur, en hiver, la chaleur» vise une atmosphère poétique à atteindre par l'harmonie infinitésimale de détails murement réfléchis dans la synthèse des arts. "Devance en chaque chose le temps » demande que tout soit planifié avec soin, souplesse et vigilance. L'exactitude, lente à maitriser, permet l'économie des moyens. "Prépare-toi à la pluie » signifie s'attendre à tout, et vise en particulier l'adaptation avec aisance à toutes les circonstances inattendues usuellement appréhendées comme « fâcheuses » : «Le but essentiel du maitre de thé est de cultiver chez l'élève la capacité d'affronter avec sérénité chaque situation, quelle qu'elle soit, le cœur ouvert et l'esprit libre et sincère » (Sen 55). "Accorde à chacun de tes invités la plus grande attention » enseigne que tant l'hôte que l'invité se doivent d'agir avec les plus grands égards l'un envers l'autre. L'hôte prépare et sert le thé avec toute son âme, l'invité doit le recevoir de la même façon, avec délicatesse, simplicité, respect, présence totale.

\section{3- Une synthèse des arts}

Le pavillon de thé traditionnel est emblématique de la cérémonie du thé, même si celle-ci se pratique aujourd'hui aussi dans des bâtiments en dur. A l'intérieur, un espace presque entièrement vide d'environ neuf mètres carrés, permettant juste d'accueillir une demi-douzaine de personnes assises. Le pavillon porte aussi l'appellation de «Maison de l'Asymétrique » et de « Maison du Vide». «Un élément vital du cha no yû est le rouleau d'inspiration zen suspendu dans l'alcôve (tokonoma) du pavillon de thé » (Addis 12). On trouve aussi au sein de cette alcôve intime dans la suite de la cérémonie un arrangement minimaliste de fleurs de saison fraichement cueillies (ou par exemple l'association épurée d'une fleur et d'une brindille) toujours parfaitement contextualisé : « un arrangement floral 
combiné par un maitre de thé perd toute sa signification si on l'enlève de l'endroit auquel il a été destiné, car toutes ses lignes, toutes ses proportions, ont été composées en vue de s'harmoniser avec les objets environnants » (Okakura 90). Cet environnement sobre et ritualisé, «neutre » et «fade » en apparence, où chaque élément esthétique est subtilement relié à un autre, quoique comportant une part d'inachevé volontaire que le jeu de l'intuition peut appréhender à sa guise, induit aussi une autre dénomination du lieu : «Maison de la Fantaisie ». Celle-ci étant conçue comme une structure éphémère construite à seule fin d'abriter une impulsion poétique de haute qualité sensible, la cérémonie du thé se révèle ainsi comme « l'art de cacher la beauté que l'on est capable de découvrir, et de suggérer celle que l’on n'ose pas révéler » (Okakura 18).

\section{2- Cérémonie du thé et arts martiaux : "la technique c'est l'homme !"}

La démarche du chanoyu relevant d'une très haute exigence, choisir d'appréhender à présent, hardiment, la cérémonie japonaise du thé sous l'angle majeur du budô (« art de la paix », « voie martiale »), du « combat sur soi », revient à l'intégrer dans une dimension philosophique plus étendue de la pensée japonaise. En effet, «les voies du budô sont des pratiques de la ritualisation de la relation qui incarnent à la fois des principes chinois et japonais d'origine multiples, majoritairement taoïstes et bouddhistes mais aussi shintoïstes, confucianistes, etc. » (Martin-Juchat 112). De ce point de vue, et même si le terme «kata» ne fait pas partie du vocabulaire traditionnel de la cérémonie du thé, la Voie du thé (chadô), elle-même incluse dans le champ de «la Voie des arts » $\left(\right.$ geïdô ${ }^{4}$, peut certainement être rapprochée d'un art martial. En effet, cha veut dire thé et $d \hat{o}$ veut dire discipline, chemin, voie conduisant vers un état d'esprit (sobrement évoqué comme «libérant les facultés humaines »). C'est aussi pourquoi les maitres enseignant les arts martiaux au Japon recommandent fréquemment à leurs élèves de pratiquer la cérémonie du thé.

En revenant quelques siècles en arrière dans un contexte féodal agité, c'est par le biais du chanoyu - fréquemment pratiqué avant la bataille - que les samouraïs « faisaient le vide» dans leur esprit. Dans le combat réglé par l'éthique exigeante du bushidô (bu-martial, shiguerrier, dô-voie), la «réalisation de soi » exigeait le dépassement de la peur et l'apprivoisement de la mort dans sa propre intimité : rester ouvert à l'intention de l'autre, sans pensées ni émotions parasites, dans la position intérieure de la «disponibilité » au présent et du «non-agir taoïste» (wu-wei), lequel permet l'action juste spontanée, donc la plus grande efficience aux combats. Comme l'écrit François Jullien, « ne rien faire (et que rien ne soit pas fait)» (109). 
Tous les arts martiaux ont comme point commun de base le modèle du «kata». Celui-ci peut être défini comme une «séquence de gestes formalisés et codifiés, sous-tendue par un état d'esprit orienté vers la réalisation de la voie» (Tokitsu 28) et qui condense une certaine expérience. Le maitre zen Taisen Deshimaru confirme que « la vraie essence des katas se retrouve non dans les gestes eux-mêmes, mais dans la façon dont l'esprit les rend justes » (54). Kenji Tokitsu affirme que « dans les arts traditionnels japonais, le but du kata est identique : réaliser, sous une forme parfaite, un ensemble de mouvements transmis par la tradition, cette perfection s'obtenant par l'union de techniques gestuelles et d'une certaine disposition d'esprit : suivre la "voie" (dô)» (31).

L'auteur ajoute que le processus de perfectionnement dans une discipline - quelle qu'elle soit - est celui de l'accomplissement de la personnalité toute entière et non pas la seule acquisition d'une compétence particulière. Plus précisément, le mot «technique »a aujourd'hui deux traductions au Japon : gi jutsu, la plus récente, adaptée de l'Occident pour désigner la technique dans la production industrielle et dans la "science de l'objet» moderne, conception limitée dont les applications fructueuses ont engendré une puissance inouïe ; et wasa, la notion traditionnelle aujourd'hui notamment confinée aux arts corporels et manuels (calligraphie, peinture, sculpture, poterie, etc.). Dans cette conception wasa, l'homme est inclus dans la technique. Comme semble le vérifier les dernières avancées des neurosciences, la réalisation, dans ce qu'elle a de décisif, se situe en un moment d'intuition où le corps et l'esprit se fondent : «l'action efficace jaillit d'une perception préconsciente et d'une décision antécédent toute conscience de volition » (Marcel 95). En ce sens, il existe un « infini technique» humain du kata, même si celui-ci n'exclut pas la réflexion logique en amont ou en aval du processus.

Ainsi, «dans la technique au sens de waza, l'homme et son geste tendent à l'indistinction mais c'est le haut degré de perfectionnement qualitatif de la réalisation qui fait sa spécificité » (Tokitsu 39). En transposant ce qui vient d'être écrit à la cérémonie du thé, il semble clair que le pôle éthique des quatre principes du chanoyu (wa-kei-sei-jaku) appartient de fait intégralement à sa technique corporelle manifestée.

Sur un plan beaucoup plus vaste, efforts et patience sont des réalités incontournables de toute voie de réalisation traditionnelle japonaise. Apprentissage de la discipline, de l'humilité, pédagogie intense de l'attention...

L'apprentissage des techniques sera donc extrêmement progressif. « Bruler les étapes » équivaudrait à incendier ses fondations... Si le pratiquant n'est pas capable de «s'ouvrir» et de « communiquer», d'être en harmonie avec luimême et avec ses partenaires alors même qu'il se borne à saluer très lentement 
[...], il lui faudra donc recommencer inlassablement son salut jusqu'à ce que l'esprit du «rei $»-$ la recherche d'unification interne et de communion avec autrui - le pénètre profondément... (Thelen 100)

Avec pour objectif lointain le scintillement du célèbre précepte zen: "faire de sa vie une œuvre d'art».

\section{3- Le chanoyu comme reliance intersubjective impersonnelle}

L'ascèse technique au sein d'une "conscience globale » (Depraz 172), et la sensibilité à l'imperceptible, permettent d'entrevoir la qualité inintelligible de relation sans intention à cultiver à travers l'expérience du chanoyu : une « attention vigilante », susceptible de conduire à l'intégration d'une perception consciente antérieure à la fragmentation du sujet et de l'objet, donc non-identifiée et détachée de la connaissance d'objet. "Cette attention vigilante, dans le Zen, est conçue comme une vacuité mentale et une adéquation directe à l'immédiat. Celui qui connait le gout du thé, enseignaient les maitres de cérémonie, c'est celui qui le boit, un point c'est tout » (Marcel 110).

Durant une cérémonie qui dure plusieurs heures dans sa version intégrale, le maitre de thé, l'hôte ou l'hôtesse de cérémonie, se doivent, dans l'échange des respirations, d'engager leur être entier pour créer une atmosphère de purification et d'intensification de la perception propice au ravissement esthétique et à la communion. Par le «non-agir» (aucune « intentionnalité » explicite : restrictive, limitative !), le Maitre de thé module la consonance de la cérémonie, laquelle vise simplement, par la beauté comme «perfection technique partagée », au relâchement complet des tensions physiques, émotionnelles et mentales.

$\mathrm{Au}$ cœur du chanoyu, pas de représentation, pas d'acteurs, ni de spectateurs: seulement une relation technique (wasa) d'ordre qualitatif, sensible et irrémédiablement unique. Selon l'expression usuelle (Sen 58) : "une fois, une rencontre » (ichigo ichie). Au cours d'une cérémonie du thé « impeccable » selon la tradition précise du chadô : «personne rencontre personne » (muhinshu). Dans cette assertion déconcertante, quoique décisive, issue du zen, « mû se réfère au néant; hin, à l'invité ou à celui qui est élevé dans les principes du zen; et shu, à l'hôte »(58). Nous rejoignons ici une problématique majeure de certains cognitivistes, selon laquelle il nous faudrait aujourd'hui éduquer notre expérience à la déconstruction totale de l'image occidentale classique du sujet humain, à même de «nous permettre, existentiellement, de nous voir comme des êtres pensants sans sujet et de faire nôtre, "sans angoisse", une éthique du "sans fond" » (Varela, Thompson et Rosch 308). 
Dans ces moments privilégiés, probablement rarissimes à leur plus haute expression collective, chacun serait physiquement présent, mais la « crispation égocentrique » identitaire aurait momentanément lâchée. Il en résulterait un sentiment de liberté inattendu et stupéfiant. La communication, au sens étymologique premier de «communion», serait alors qualitativement parfaite entre les êtres. Dans cette dimension anthropologique particulière de la conscience, affirmée dans le zen comme plus profonde que celle de la pensée, chaque forme resterait séparée, non comparable parce que irréductiblement différente (voir Bouchez $)^{6}$, mais la « banalité des choses » cognitivement généré par le « regard obscurci », « encombré » et « quasi-somnambulique » de l'adulte, ne se serait révélée être rien de plus qu'une illusion. Tout serait, dès lors, (re)devenu «beau, rare et précieux » pour ceux qui feraient l'expérience de gouter, ensemble, et à des degrés divers, la fraicheur vivifiante de cette perception intuitive replongeant aux sources de la virginité enfantine. L'ensemble codifié des «katas» de l'étiquette de la cérémonie du thé s'inscrirait alors dans un «écrin naturel d'éternité » comme une « fête des sens » toujours renouvelée.

\section{Conclusion : de l'urgence d'une éducation occidentale à la présence ?}

L'Occident (moderne) commence-t-il à mieux comprendre l'Orient (traditionnel) ? Après la catastrophe de Fukushima (2011) qui cristallise peut-être aujourd'hui au Japon la prise de conscience des limites de l'hyper-rationalisation et de la technique dans son mode gi jutsu, l'exemple singulier du chanoyu peut certainement contribuer à «élargir » le champ de réflexion sur les TICE quant à l'optimisation qualitative, dans l'espace interculturel, de la mise en réseaux croissante des intelligences éducatives et des savoirs.

Nous avons montré que l'art du chanoyu, si complexe et pourtant si simple, par sa beauté habile et pénétrante, par la profondeur et l'efficience apportées à chaque action, par l'harmonie esthétique et le climat de pacification reliante qu'il vise à instituer entre tous les participants, constitue une entrée majeure dans les « fondements techniques » (dans son mode wasa) de l'une des principales cultures asiatiques, et qu'il accroit les capacités de l'individu à agir sur soi et avec les autres. Parfaitement contextualisé localement mais aussi universel de part ses «finalités techniques », l'exemple extrême de l'expérience du chanoyu nous confirme aussi sans ambigüité possible, au terme de notre parcours, que la cognition humaine n'est nullement « compressable ».

Aujourd'hui, les TICE au service des arts, de l'apprentissage des langues et des cultures sont des inventions enthousiasmantes. Cependant, cette virtualisation de l'intelligence éducative interculturelle n'apparait pertinente que si, dans une dialogique adéquate, l'ancrage physique 
dans le réel est cultivé, discipliné, et affiné. En ce sens, le développement de techniques d' « éducation à la présence » semble à même, en particulier dans les pays à haute densité numérique, de compenser l'impact dommageable sur l'être humain des réseaux ubiquitaires et autres dispositifs de réalité virtuelle comme « systèmes de communication sans le corps ». Et d'arriver à corriger des déséquilibres «discrets» (regarder sans voir, écouter sans entendre, sentir sans ressentir...) en plein essor liés à l'appauvrissement «qualitatif» vertigineux de la perception sensorielle d'un individu, «hypermoderne» d'un côté, « primitif» (Debray 393) voire « infirme » de l'autre.

Comme l'écrit Bernard Bachelet à propos des stoïciens, "Être éveillé, être vigilant actuellement, voilà le seul bonheur qui ne soit pas fantasmatique » (245). Apprentissage laïcisé, discipliné et puissamment motivé, tant au niveau individuel que sociétal, de «l'art d'être soi », totalement disponible à la densité du moment présent. L'expérience incarnée du chanoyu pointant la nécessité d'une possible et démocratique «éducation humaine à la présence », il ne tient qu'à nous en retour de souhaiter élever cette dernière en tête de nos priorités, en inventant et en développant sous mille formes pertinentes dans nos activités éducatives et quotidiennes des « techniques » particulières de mise en pratique.

\section{Bibliographie}

Addis, Stephen. L'art zen. Paris : Bordas, 1992.

Bachelet, Bernard. Sur quelques figures du temps. Paris : Vrin, 1996.

Bouchez, Pascal. « De quelques prérequis nécessaires à l'émergence de fluidités individuelles et collectives pertinentes en contexte multiculturel d'apprentissage socio-numérique ». Tic et construction du lien social dans la multiculturalité. TICEMED 2011. Barcelone, 8-10/06/2011. Actes en ligne :

http://www.ticemedconf.org/home/index.php?option=com_content\&view=category\&l ayout $=$ blog\&id $=62 \&$ Itemid $=105 \&$ lang $=f r$

Bui-Xuân, Gilles et Sébastien Ruffié. «Le salut en judo: un médium ritualisé de communication ». Corps et culture (1999). http://corpsetculture.revues.org/564 [page consultée le 05/08/2012].

Debray, Régis. Vie et mort de l'image. Une histoire du regard en Occident. Paris : Gallimard, 1992.

De Peretti, André. «Rogers et Krishnamurti : une rencontre intellectuelle». Symposium Krishnamurti et l'éducation à la fin du XXe siècle 29-30/05/1995, Paris 8. 
http://www.barbier-rd.nom.fr/rogersetkrishnamurti.htm [page consultée le 24/11/2011].

Depraz, Nathalie. La conscience. Approches croisées des classiques aux sciences cognitives.

Paris : Armand Colin/Vuef, 2001.

Deshimaru, Taisen. Zen \& arts martiaux. Paris : Seghers, 1977.

Jullien, François. Traité de l'efficacité. Paris : Grasset \& Fasquelles, 1996.

Okakura, Kakusô. Le Livre du thé. Paris : Payot \& Rivages, 2004 ( $1^{\mathrm{e}}$ édition anglaise 1906).

Martin-Juchat, Fabienne. «La notion d'intercorporéité dans les arts martiaux orientaux ». Médiations du corps. Grenoble : Gresec, 2000.

Maisonneuve, Jean. Les conduites rituelles. Paris : PUF, 1999.

Marcel, Antoine. Zen et connaissance. Escalquens : Oxus, 2009.

Reed, William. Le Ki. Paris: Trédaniel, 1994 ( $1^{\mathrm{e}}$ édition en langue anglaise, Ki, Japon Publications, Tokyo et New York, 1986).

Sen Sôshitsu. Vie du thé, esprit du thé. Paris : Seld / Godefroy, 1994.

Shimizu, Christine., dir. Les Arts de la Cérémonie du Thé. Dijon : Faton, 1996.

Thelen, Yves. Budo. Retour aux Sources. Paris : Tredaniel, 1992.

Tokitsu, Kenji. Les Katas. Méolans-Revel : Désiris, 2002.

Varela, Francisco, Evan Thompson et Eleanor Rosch. L'inscription corporelle de l'esprit. Sciences cognitives et expérience humaine. Paris : Seuil, 1993.

Watts, Alan. Amour et connaissance. Paris : Denoël/Gonthier, 1971 ( $1^{\mathrm{e}}$ édition Pantheon, New York, 1958).

\footnotetext{
NOTES

${ }^{1}$ C'est l'objet exclusif de nombreux traités spécialisés. Cf. la bibliographie de l'ouvrage Les Arts de la Cérémonie du Thé (Shimizu 252-253), pour un aperçu de la production dans ce domaine.

${ }^{2}$ Dans l'optique relationnelle «non-directive » et « qualitative » chère à Carl Rogers, André de Peretti a insisté dans plusieurs de ses travaux sur « la présence à soi-même sans tension, sans contradiction, et surtout sans projet de défensivité ».

${ }^{3}$ Cette «économie d'énergie » vise notamment à neutraliser peu à peu l'attitude de crispation habituelle inconsciente de tout un chacun, laquelle s'exprime par «l'habitude d'employer beaucoup plus d'énergie que nécessaire pour penser, voir, entendre, ou prendre une décision » (Watts 98).

${ }^{4}$ Calligraphie, musique, danse, poésie, arrangement floral, etc.

5 « A cet égard, la conception de la corporéité est située sur un plan à la fois individuel et universel, qu'il soit cosmologique ou ontologique, qui renvoie dans tous les cas de figure à une conscience globale excédant l'individu. »

6 J'ai proposé le concept de "distanciation empathique» pour rendre compte d'un tel processus communicationnel "fluidifié », lequel requiert d'une part une attention distanciatrice en temps réel envers ses perceptions et représentations, d'autre part l'apprentissage patient d'une empathie consciente vis-à-vis de soi et des autres, perçus en dialogique verticale comme tout à la fois totalement « autres » (« incommunicabilité ») et indissociablement reliés (« compréhension »).
} 